complete depletion of peripheral B-cells, memory and naïve Bcell subsets, and plasmablasts, with $89 \%$ of obinutuzumab patients depleted to $<0.441 \mathrm{CD} 19+$ cells $/ \mu \mathrm{L}$ at week 4 . Among obinutuzumab patients, sustained B-cell depletion was associated with greater renal response at week 76 (table 1), although patients who achieved sustained depletion also had lower baseline proteinuria and serum creatinine.

Conclusions Obinutuzumab, a type II anti-CD20 mAb, induced rapid and complete depletion of peripheral B-cells and B-cell subsets. Similar to previous reports, sustained Bcell depletion was associated with increased renal response. Further evaluation is ongoing to understand the factors associated with achievement of sustained B-cell depletion and renal response.

Acknowledgements This study was funded by F. Hoffmann-La Roche.

\section{LOW-DOSE IL-2 THERAPY MODULATES LYMPHOCYTE SUBSETS THAT ARE INVOLVED IN THE REGULATION OF GERMINAL-CENTRE REACTIONS IN PATIENTS WITH SLE}

${ }^{1,2}$ Jens Y Humrich, ${ }^{1}$ Caroline von Spee-Mayer, ${ }^{1}$ Elise Siegert, ${ }^{1}$ Martina Bertolo,
${ }^{1}$ Angelika Rose, ${ }^{1}$ Philipp Enghard, ${ }^{1}$ Falk Hiepe, ${ }^{1}$ Tobias Alexander, ${ }^{1}$ Gerd-
Rüdiger Burmester, ${ }^{1,2}$ Gabriela Riemekasten. ${ }^{1}$ Dept. of Rheumatology and Clinical
Immunology, Charité - University Medicine Berlin, Berlin; ${ }^{2}$ Dept. of Rheumatology and
Clinical Immunology, University Hospital Schleswig-Holstein - Campus Lübeck, Lübeck,
Germany

10.1136/lupus-2020-eurolupus. 47

Background Low-dose IL-2 therapy was shown to restore regulatory $\mathrm{T}$ cell (Treg) homeostasis and to decrease disease activity in early phase clinical trials in patients with SLE. ${ }^{1,2,3}$ However, the mechanisms of action of IL-2 therapy beyond Treg targeting are still poorly understood. Here, we assessed the changes of lymphocyte subsets in the peripheral blood that are considered to be involved in the regulation of germinal-center reactions during an open-label, uncontrolled, phase $1 / 2$ a single-center trial with low-dose IL-2 therapy in patients with active SLE.

Methods 12 patients with active and refractory SLE (SLEDAI $\geq 6$ ) were treated with four separate 5-day treatment cycles consisting of daily subcutaneous injections of IL-2 between 0.75 and 3.0 million IU. Cells from peripheral blood were analyzed by flow cytometry before and one day after each treatment cycle.

Results Significant decreases in the numbers of CD3-CD56CD19+ B cells were observed after most treatment cycles and at the end of the treatment period. Subtyping of the B cell population revealed that in particular proportions and numbers of $\mathrm{CD} 20+\mathrm{IgD}+\mathrm{CD} 27+$ marginal-zone $\mathrm{B}$ cells were reduced. Transient and moderate decreases in the proportions and numbers of CD20+IgD-CD27- memory B cells were also apparent during the treatment cycles, whereas there were no relevant changes in $C D 20+\operatorname{IgD}+\mathrm{CD} 27$ - naïve $\mathrm{B}$ cells. In addition we noted moderate decreases in the proportions of common CXCR5 $+\mathrm{T}$ follicular helper cells (Tfh) and of CD45RO+CCR7-CXCR5+PD1+ Tfh cells among $\mathrm{CD} 3+\mathrm{CD} 4+$ FoxP3 $-\mathrm{T}$ cells during the treatment period. Yet, more pronounced decreases were observed in the proportions of CXCR5+ cells among CD3+CD4 + FoxP3 + regulatory $\mathrm{T}$ cells.
Conclusions These findings suggest that low-dose IL-2 therapy interferes with the regulation and fine tuning of immune responses that take place in the germinal-centres of lymphatic tissues.

Funding German Research Foundation (DFG).

\section{REFERENCES}

1. Humrich JY, von Spee-Mayer C, Siegert E, Alexander T, Hiepe F, Radbruch A, Burmester GR, Riemekasten G. Rapid induction of clinical remission by lowdose interleukin-2 in a patient with refractory SLE. Ann Rheum Dis. 2015; 74; 791-792.

2. von Spee-Mayer $C$, Siegert E, Abdirama D, Rose A, Klaus A, Alexander T, Enghard P, Sawitzki B, Hiepe F, Radbruch A, et al. Low-dose interleukin-2 selectively corrects regulatory $T$ cell defects in patients with systemic lupus erythematosus. Ann Rheum Dis. 2016; 75; 1407-1415.

3. Humrich JY, von Spee-Mayer $C$, Siegert $E$, Bertolo $M$, Rose $A$, Abdirama $D$, Enghard $P$, Stuhlmüller B, Sawitzki B, Huscher D, et al. Low-dose interleukin-2 therapy in refractory systemic lupus erythematosus: an investigator-initiated, single-centre phase 1 and 2a clinical trial. Lancet Rheumatol. 2019; 1; e44-e54.

\section{M5049, A NOVEL POTENT AND SELECTIVE INHIBITOR OF TOLL-LIKE RECEPTORS 7 AND 8 (TLR 7/8)}

${ }^{1}$ Brian Sherer, ${ }^{2}$ Andrew T Bender, ${ }^{2}$ Albertina Pereira, ${ }^{3}$ Sonja Reissig, ${ }^{3}$ Philipp Haselmayer, ${ }^{2}$ Shinji L Okitsu, ${ }^{2}$ Evgeni Tzvetkov, ${ }^{2}$ Melinda Przetak, ${ }^{2}$ Noune Tahmassian Morse, ${ }^{2}$ Jaromir Vlach. 'US Chemistry, EMD Serono*, Billerica; ${ }^{2}$ TIP Immunology, EMD Serono*, Billerica, USA; ${ }^{3} T I P$ Immunology, Merck Healthcare KGaA, Darmstadt, Germany; *A business of Merck KGA, Darmstadt, Germany

\subsection{6/lupus-2020-eurolupus.48}

Background TLR 7/8 are transmembrane receptors that recognize single-stranded RNA. In humans, TLR7 is mainly expressed in B cells and plasmacytoid dendritic cells and its activation stimulates antibody production and secretion of cytokines including type I interferon (IFN). Activation of TLR8 in myeloid cells leads to cytokine production, and activation of antibacterial host-protective mechanisms (eg, neutrophil NETosis). Aberrant activation of TLR $7 / 8$ is potentially pathogenic and linked to progression of certain autoimmune diseases such as SLE. Thus, inhibition of TLR7/8 may be an effective treatment approach for SLE.

Methods Compounds were prepared and optimized for potency by testing them in TLR7/8-stimulated HEK 293 cells, peripheral blood mononuclear cells (PBMCs), and human whole blood. M5049 was selected as the compound with the best balance of potency, pharmacokinetic, and physiochemical properties. The pharmacokinetic and pharmacodynamic activity of M5049 was assessed in mice to determine its in vivo potency and duration of action. M5049 was also tested in mouse lupus models.

Results M5049 showed potent and selective activity in a wide range of cellular assays for inhibition of TLR7/8. M5049 inhibited R848-stimulated cytokine production in human PBMCs and whole blood, and TLR8-mediated NETosis in human primary neutrophils. In mice, M5049 showed a dose-dependent inhibition of R848-stimulated cytokine release and a long duration of action. M5049 demonstrated efficacy in suppressing disease development in the BXSB-Yaa and IFN- $\alpha$ accelerated NZB/W F1 mouse models of lupus.

Conclusions M5049 is a highly potent and selective inhibitor of TLR 7/8 that effectively reduces lupus pathogenesis in mice. M5049 may have potential to treat autoimmune diseases such as SLE in humans. 